\title{
Spectroscopic diagnostic for the ring-size of carbohydrates in the gas phase: furanose and pyranose forms of GalNAc
}

\author{
Baptiste Schindler, a Laurent Legentil, b Abdul-Rhaman Allouche,a Vincent Ferrières* ${ }^{\star}$ and Isabelle \\ Compagnon * ${ }^{a c}$
}

Hexoses are mainly found in nature in the pyranose form (6-membered ring). Yet, furanose forms (5-membered ring) are observed in some rare polysaccharides. Using IRMPD spectroscopy (InfraRed Multiple Photon Dissociation), we propose a straightforward diagnostic of the ring-size of $\mathrm{N}$-acetyl galactosamine ions. The furanose form of $\mathrm{N}$-acetyl galactosamine was synthesized and its protonated ion was isolated in an ion trap to measure its gas phase vibrational spectrum by IRMPD. Comparison with the IRMPD spectrum of its pyranose counterpart reveals that they have distinctive optical fingerprints. This new MS-based diagnostic opens the way to facile identification of the ring-size in oligosaccharides. Our experimental data also provide new insights to support the theoretical description of the conformational behavior of the furanose ring, which is notoriously more flexible than the pyranose form but remains difficult to assess.

\footnotetext{
1 a Univ. Lyon, Université Claude Bernard Lyon 1, CNRS, Institut Lumière Matière, F-69622 Villeurbanne, France. E-mail: isabelle.compagnon@univ-lyon1.fr

b Univ. Rennes, Ecole Nationale Supérieure de Chimie de Rennes, CNRS, UMR 6226 - ISCR, F-35 000 Rennes, France. E-mail: vincent.ferrieres@ensc-rennes.fr

Institut Universitaire de France IUF, 103 Boulevard St Michel, Paris F-75005, France

$\dagger$ Electronic supplementary information (ESI) available: Additional computed IR spectra of conformers of b-D-Galf NAc-OMe; molecular drawings of conformers with hydrogen bonds; sorted energy values of the 608 calculated structures; NMR spectra.
} 


\section{Introduction}

$\mathrm{N}$-Acetyl galactosamine (GalNAc) is a widespread carbohydrate in Nature. As for all hexoses, it is generally found in the 6-membered ring pyranose form (GalpNAc), which is less constrained and thus energetically more favorable than its five-membered ring furanose counterpart (Galf NAc). Yet, the furanose form is observed in rare polysaccharides from bacterial sources. 1

GalpNAc is found in numerous glycoconjugates such as glycosphingolipids which represent a highly heterogeneous family of biomolecules mainly present in brain of vertebrates.GalNAc is also found in glycoproteins.2 In mucine, the residue of interest is linked, through an a-O-glycosidic linkage, to serine or threonine. This simple glycosyl amino acid is best known as Tn antigen. Nevertheless, further stretching of the carbohydrate chain results in high molecular diversity, yielding O-glycans, as well as high biological complexity.3 The GalNAc monosaccharide is also found in glycosaminoglycans, and more especially in proteoglycans. While $\mathrm{N}$-acetyl glucosamine (GlcNAc) is related to the family of heparan sulfates, chondroitin sulfates are formed with GalNAc residue.4 Both families of charged polysaccharides are still widely studied for their complex structures and for their potential applications in cosmetics and medicine. Moreover, since GalNAc is an excellent ligand of a sialoglycoprotein receptor, it was also used to deliver bioactive antisense oligonucleotides in liver through interaction with membrane hepatocytes.5

On the other hand, some rare polysaccharides analyzed by NMR spectroscopy have been found to contain GalNAc in a furanose form. For instance, the O-specific polysaccharide produced by Proteus penneri strain 22, responsible for urinary tract infections with subsequent complications, presents a Galf NAc residue linked to the 4-position of a glucuronic acid entity. 6 Moreover, the Gram negative bacteria Campylobacter jejuni 11168, responsible for serious gastroenteritis, is able to biosynthesize a chain (containing - 2)-b-D-Ribf-(1 - 5)-b-DGalf NAc-(1 - 4)-a-D-GlcpA.7

Why would Nature occasionally choose to biosynthesise carbohydrates in the furanose form over the more energetically favorable pyranose form? The biological role of furanose residues is still unclear. Extensive conformational exploration of furanose rings and furanose-containing oligosaccharides reported by Lowary et al. have shown that five-membered carbohydrate rings are significantly more flexible than sixmembered rings.8,9 Yet, the conformation of the furanose form of hexoses remains a matter of debate. Their particular conformational behavior however may be responsible for unique biological functions.

Gas phase spectroscopy, in combination with quantum chemistry simulations, is an established method for the exploration of the intrinsic conformational preferences of biomolecules.10 Such approach was first applied to phenyl-grafted carbohydrates, 11 including $\mathrm{N}$-acetyl hexosamines 12 by Prof. John Simons. Alternative spectroscopic schemes such as rotational spectroscopy in supersonic jet expansion;13,14 helium droplet spectroscopy; 15

cryogenic spectroscopy;16 and IRMPD spectroscopy (infraredmultiple photon dissociation spectroscopy) 17 have opened the way to systematic analysis of natural carbohydrates.

The conformation of GalNAc ions in the gas phase has been investigated using IRMPD spectroscopy and Density Functional Theory (DFT) on protonated ions;18,19 lithium adducts;20,21 and sodium adducts.22 Regardless of the charge state, a ${ }_{4} \mathrm{C}_{1}$ conformation of the pyranose ring was reported as the most stable structure, which is consistent with the conformation of pyranose structures in the condensed phase. For protonated GalpNAc, a relatively simple potential energy surface was found, only populated by ${ }_{4} \mathrm{C}_{1}$ conformations with slight differences in the hydroxyl coordination pattern within $20 \mathrm{~kJ}$ mol_1 of the minimum (zero point energy-corrected at the CAMB3LYP/6$311++G(2 d f, 2 p d)$ level of theory). Note that traces of Galf NAc - potentially present in the GalNAc sample in minor amount - were not detected in these studies.

More surprisingly ribose - a pentose well known for adopting the furanose form in RNA;23 and fructose a hexose locked in the furanose form in saccharose (table sugar), were only observed in the pyranose form when isolated in the gas phase.24,25 Cocinero et al. first observed free ribose in the furanose form by means of methylation of the anomeric hydroxyl group, which hinders the ring opening and the interference with pyranose signal.26 Gas phase spectroscopy of hexoses in the furanose form have never been reported, however. Here we adopt the methylation strategy proposed by Cocinero to isolate GalNAc in the furanose form and we report its IRMPD fingerprint. Comparison with the pyranose form establishes that IRMPD constitute a straightforward MS-based diagnostic of the ringsize. The conformational complexity of the furanose ring is explored using Molecular Dynamics and Density Functional Theory.

\section{Methods}

Choice of the model monosaccharides In order to select a relevant monosaccharide standard in the context of the sequencing of GalNAc-containing glycans, two hypothesis were made. Firstly, to clearly identify specific spectroscopic signatures for the furanosyl entity without any superimposition with characteristic signals for the pyranoside counterpart, we chose chemically blocked methyl Nacetylgalactosides (1: Galf NAc-OMe, 2: GalpNAcOMe; Scheme 1). Secondly, Since the Galf NAc was exclusively found in natural conjugates with the b-anomeric configuration, both targets were chosen with the 1,2-trans orientation, as shown in Scheme 1. 


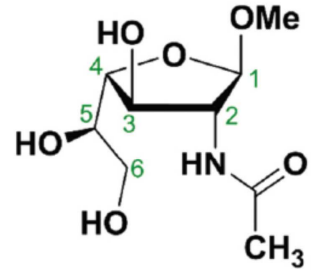

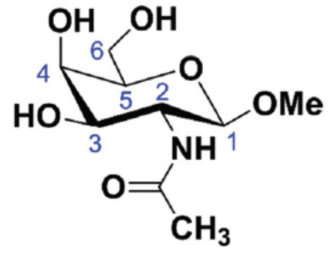

GalfNAc-OMe (1)
GalpNAc-OMe (1)

Scheme 1 Structures of compounds of interest.

\section{Samples preparation}

Monosaccharide 2 is commercially available, it was purchased from Carbosynth. The furanoside 1 had to be synthesized. The synthetic procedure is described in $\mathrm{ESI} . \dagger \mathrm{A}$ solution of 1 or 2 was prepared at a concentration of $10 \mathrm{mM}$ in water/methanol (50/50). $0.1 \%$ of acetic acid was added to stabilize the signal and to promote protonation of the ions. The solution is loaded in the electrospray ion source by direct infusion for subsequent MS, MS/MS and IRMPD analysis.

\section{IRMPD spectroscopy}

The IRMPD apparatus shown in Fig. 1 consists of a commercial mass spectrometer equipped with an electrospray ion source and a 3D ion trap mass analyzer (Thermo Scientific LCQ Classic) modified to allow irradiation of the trapped ions by a tunable YAG-pumped OPO/OPA IR laser system (LaserVision) operating at $10 \mathrm{~Hz}$. The pulse duration is $10 \mathrm{~ns}$ and the pulse energy is $3 \mathrm{~mJ}$ through the spectral range. After mass-selection of the species of interest (here $\mathrm{m} / \mathrm{z} 236$ ), the ions are stored in the trap for $800 \mathrm{~ms}$ and irradiated. The basic principle of IRMPD spectroscopy is the following: should the IR photons be resonant with a vibrational transition of the molecule, the internal energy of the ion increases, causing photofragmentation (here $\mathrm{m} / \mathrm{z}$ 204). On the contrary, upon irradiation at a nonresonant wavelength no photofragmentation will occur. After photoactivation, the resulting MS/MS spectrum is recorded.

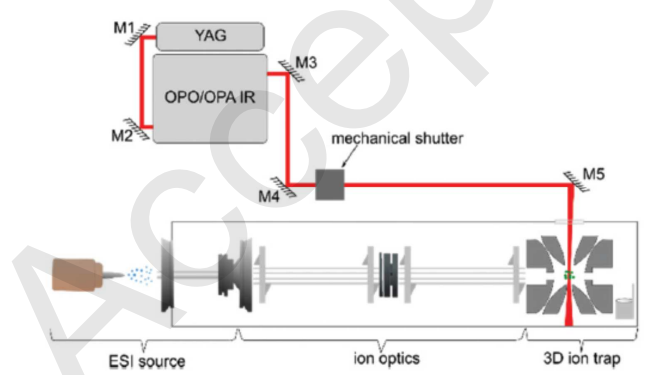

Fig. 1 Schematic of the mass spectrometer modified for integrating IR spectroscopy: a commercial LCQ Thermofinnigan mass spectrometer equipped with an ESI ion source was modified to allow laser irradiation of the ion cloud of mass selected analyses inside of the $3 \mathrm{D}$ ion trap. The IR laser beam produced by a YAG-pumped OPO/OPA laser system is guided towards the center of the ion trap, which was drilled for this purpose, using mirrors M3 to M5, and through

an IR-transparent window. A mechanical shutter is used for the synchronization of the laser excitation with the period of isolation of the ions inside the trap.

The photofragmentation yield is derived from the mass spectrum using an equivalent of the BeerLambert formula (1):

Yield $=-\log (P /(P+F))(1)$

with $P$ the intensity of parent ion and $F$ the summed intensity of each photofragment. This MS/MS sequence based on photoactivation is repeated as the laser wavelength is continuously scanned in the 2700-3700 cm_1 spectral region at a speed of 0.4 $\mathrm{cm}_{-} 1 \mathrm{~S}_{-} 1$. Finally the IR spectrum is obtained by plotting the photofragmentation yield as a function of the wavenumber. The spectra are not further corrected for wavelength-dependent power variations.

\section{Quantum chemistry}

Molecular dynamics was used to explore the potential energy surface of the protonated ion with the PM7 potential in OpenMopac.27 5 trajectories of 20 ps at $3000 \mathrm{~K}$ were ran and yielded 2000 geometries each. After optimization with PM7 and elimination of the identical conformers, 3000 geometries were obtained. At this stage, the conformations were plotted in Fig. 2a as dots on a phase amplitude conformational wheel (a color code is used to indicate their relative energy) to verify that the conformational space was thoroughly explored. The geometries were further optimized with three steps of Density Functional Theory (DFT): firstly B3LYP $28-30 / 6-31 G^{*}$, then CAM-B3LYP ${ }_{31} / 6-31 G^{*}$, and finally CAM-B3LYP/6-311++G(2df,2pd).32-34 This resulted in the reduction of the number of stable geometries (608 conformations). These conformations were plotted on a phase amplitude conformational wheel in Fig. $2 \mathrm{~b}$. The energies were further refined by single point calculations with MP235/6-311++G(3df,3pd). At this stage, the 30 lowest energy conformers were selected for computing harmonic frequencies with CAMB3LYP/6-311++G(2df,2pd). An empirical scaling factor of 0.94736 was used for comparison with the experimental IRMPD spectra. The mode analysis was performed using Gabedit,37 Gaussian0938 was used for DFT calculations and Orca3s was used for MP2 single-point energies.

\section{Results and discussions}

MS analysis and tandem MS analysis TheMS data are shown in Fig. S11 (ESI $\dagger$ ). Both GalNAc forms are detected by mass spectrometry (MS) in their protonated charge state at $236 \mathrm{~m} / \mathrm{z}$. Under collisional activation (MS/MS), they both yield a single fragment at $204 \mathrm{~m} / \mathrm{z}$, which corresponds to the loss of a neutral methanol group from C1. Note that the fragmentation yield is superior for the pyranose form. The exact same fragments are observed under photoactivation (not shown). 

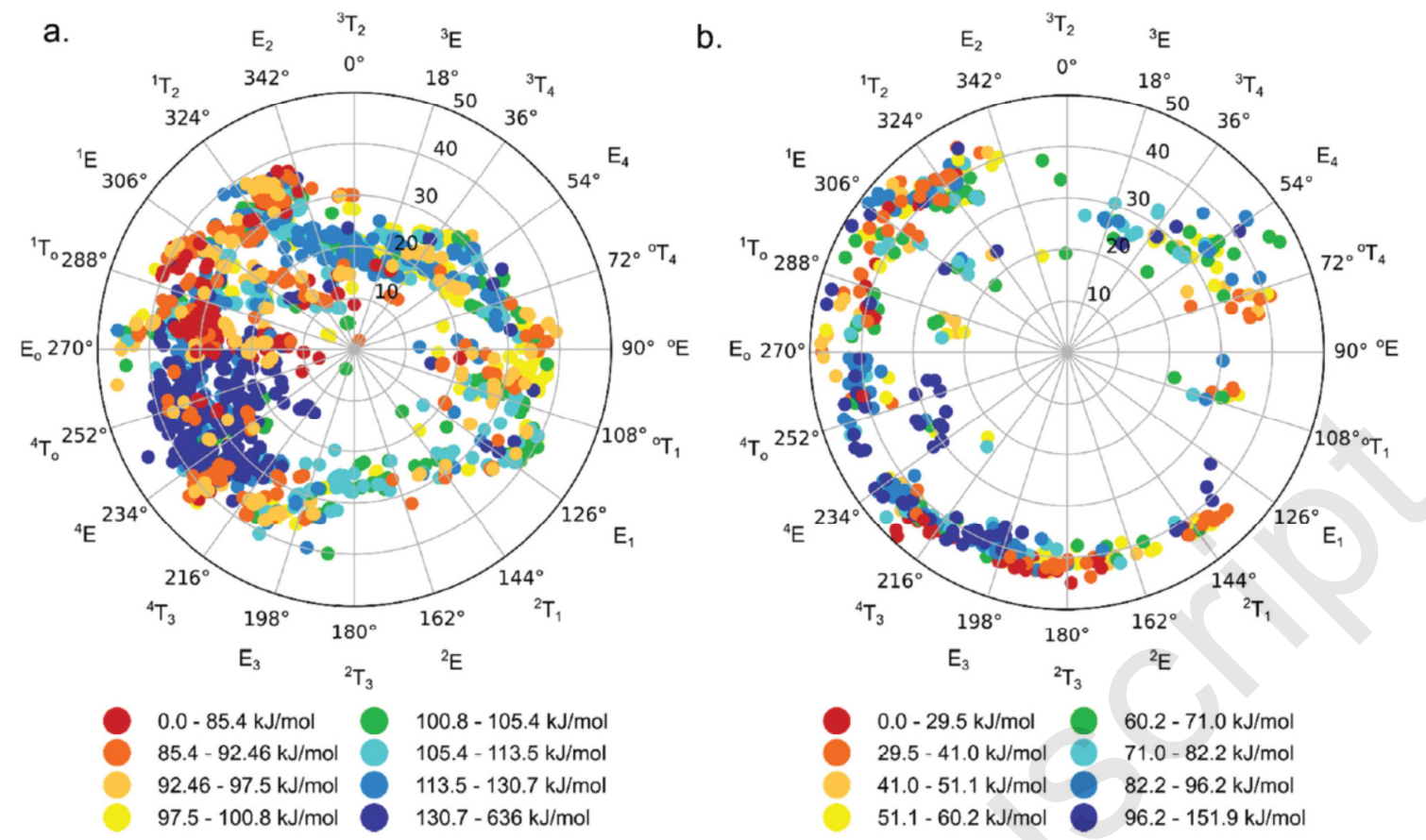

Fig. 2 (a) Coordinates of the 3000 MOPAC structures on a phase/amplitude conformational wheel. (b) Coordinates of the 608 structures after optimization at the CAM-B3LYP/6-31G* level of theory on a phase/amplitude conformational wheel.

\section{IRMPD analysis}

The IRMPD spectrum of protonated b-D-Galf NAc$\mathrm{OMe}$ is shown in Fig. 3 along with one previously reported for b-D-GalpNAc-OMe.18 Both spectra consist of three main regions of vibrational activity: the $\mathrm{CH}$ stretching modes below $3000 \mathrm{~cm}_{-} 1$, an intense feature corresponding to the $\mathrm{NH}$ stretching mode at $3375 \mathrm{~cm}_{-}$, and the $\mathrm{OH}$ stretching modes over $3400 \mathrm{~cm} \_1_{1}$. In the latest region, broad features at lower wavenumbers correspond to $\mathrm{H}$-bonded hydroxyl groups, while free or weakly coordinated hydroxyl groups appear as a narrow band at higher wavenumbers. In spite of these shared features, the two spectra are distinctive. Overall, the photofragmentation yield is higher for pyranose than for furanose in identical experimental conditions. $b$ D-Galf NAc-OMe shows a simple $\mathrm{CH}$ pattern with only two visible bands at 2910 and $2950 \mathrm{~cm} \_1$ while the one of b-D-GalpNAc-OMe is more complex with six bands between 2800 and $3050 \mathrm{~cm} \_1$. b-D-Galf $\mathrm{NAc}-\mathrm{OMe}$ shows a $\mathrm{NH}$ band at $3375 \mathrm{~cm}+$ (FWHM $=$ $54 \mathrm{~cm}_{1}$ ) which is slightly blue shifted and significantly broader than for b-D-GalpNAc-OMe (observed at $3366 \mathrm{~cm}_{1}, \mathrm{HWFM}=31 \mathrm{~cm} \_$). Finally the coordinated $\mathrm{OH}$ region consists of a single unresolved band centered around $3500 \mathrm{~cm}_{-} 1$ while the spectrum of b-D-GalpNAc-OMe shows two partially resolved bands at 3425 and $3475 \mathrm{~cm}_{1}$. IRMPD spectroscopy was previously used to distinguish between GalpNAc from other HexpNAc.18 Here it is established that IRMPD diagnostic offers sufficient structural resolution to further distinguish between the furanose and pyranose forms of GalNAc ions in the gas phase.

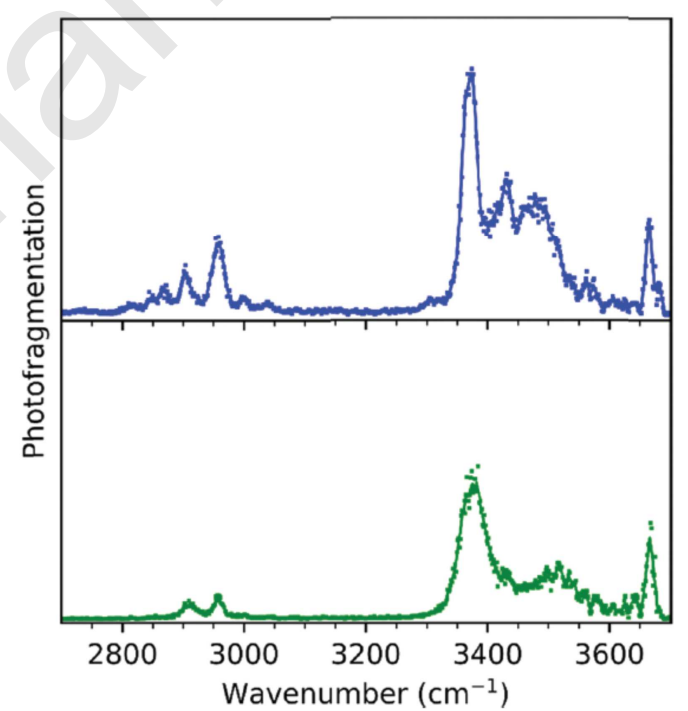

Fig. 3 IRMPD spectra of b-D-GalfNAc-OMe (green) and b-DGalpNAc-OMe (blue)

\section{Conformational analysis}

At the CAM-B3LYP/6-31G* level of theory, 608 conformers were generated. The $p$ value (pseudorotational phase angle) was calculated for each structure and is reported in Fig. 4a. The $p$ value ranges from 0 to 3601 , which validates that the conformational search resulted in a thorough exploration of the conformational space. The statistical distribution shows two main populations centered around $p=2101$ and $p=3201$, which corresponds to the South-West and North-West regions of the pseudorotational wheel. The 30 lowest energy structures are selected and reoptimized at the CAM-B3LYP/6-311++G(2df,2pd) (this criterion corresponds roughly to a $20 \mathrm{~kJ}$ mol 1 energy cut-off at the MP2 level of theory). After 
elimination of the identical conformers, 23 structures are obtained. $T$ (Twist) and $E$ (Envelope) forms are classified according to their phase angle on the phase/amplitude conformational wheel, as defined by Altona and Sundaralingam. 40 As seen in Fig. $4 \mathrm{~b}$ a first group falls in the South-South/West region (types $4 \mathrm{~T}_{3} / \mathrm{E}_{3} / 2 \mathrm{~T}_{3} / 2 \mathrm{E}$ ) and a second group falls in the North-West region (types $1 \mathrm{~T}_{2} / 1 \mathrm{E} / 1 \mathrm{To}$ ). This diversity of structures contrasts with the pyranose form, which exhibits a very simple potential energy surface, only populated by ${ }_{4} \mathrm{C}_{1}$ conformations within 20 kJ mol_1 of the global minimum.18 This observation confirms that furanose rings in the gas phase have a greater conformational flexibility than pyranose rings, and is consistent with the findings of Lowary et al. in the condensed phase. 9
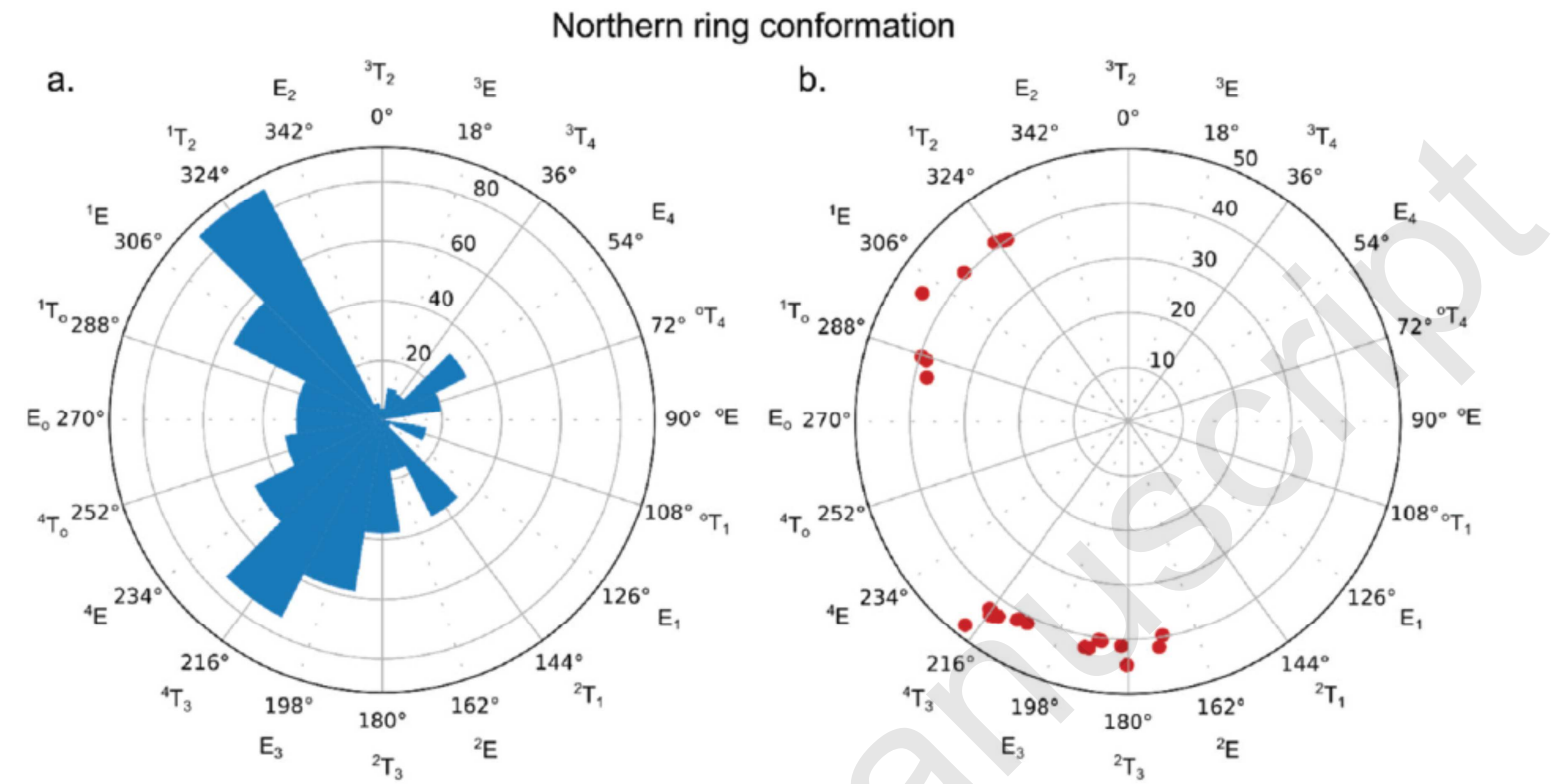

Southern ring conformation

Fig. 4 (a) Statistical distribution of $p$ value of the 608 conformers of $b$-D-GalfNAc-OMe obtained at the CAM-B3LYP/6-31G* level of theory. (b) Coordinates of the 23 lowest energy structures after re-optimization at the CAM-B3LYP/6-311++G(2df,2pd) level of theory on a phase/amplitude conformational wheel.

Since the IR spectrum in the gas phase strongly depends on both the conformation and the coordination of the hydroxyl groups, the stable conformations found within $20 \mathrm{~kJ}$ mol_1 are further sorted in sub-families according to their $\mathrm{H}$-bond network. For the South conformers, four subfamilies were identified: (i) SA6: the acetyl $\mathrm{OH}$ points clockwise and initiates the following $\mathrm{H}$-bond network: $\mathrm{Ac}(\mathrm{OH})_{\ldots}{ }_{2} \mathrm{O}_{3} \mathrm{H}_{2} \ldots$ O66; (ii) SA5: acetyl $\mathrm{OH}$ points clockwise and initiates the following $\mathrm{H}$ bond network: $\mathrm{Ac}(\mathrm{OH}) \quad \mathrm{O} 3 \mathrm{H} \quad \mathrm{O} 5$; (iii) SN6: the acetyl $\mathrm{OH}$ points counterclockwise and is $\mathrm{H}$ bonded to $\mathrm{O} 1$; and $\mathrm{O} 3 \mathrm{H}$ is $\mathrm{H}$-bonded to $\mathrm{O} 6$; and (iv) SN5: the acetyl $\mathrm{OH}$ points counterclockwise and is $\mathrm{H}$-bonded to $\mathrm{O} 1$; and $\mathrm{O} 3 \mathrm{H}$ is $\mathrm{H}$-bonded to $\mathrm{O} 5$.

For the North-West conformation, two sub-families were identified: (i) NWA: the $\mathrm{NH}$ group is free; and (ii) NWN: the $\mathrm{NH}$ group is involved in a coordination. The lowest energy structure found for each subfamily is reported in Fig. 5. Additional computed IR spectra are shown in Fig. S1 and S2 in ESI. $\dagger$ The theoretical $\mathrm{OH}$ fingerprint is strongly conformer dependent, but the $\mathrm{NH}$ frequency is almost binary: around $3425 \mathrm{~cm} \_1$ (except for NWN, where the $\mathrm{NH}$ group is coordinated with a neighbouring oxygen). Note that the match with the experimental $\mathrm{NH}$ frequency (3375 cm_1) is surprisingly poor. For comparison, the $\mathrm{NH}$ frequency of the pyranose form is observed at $3366 \mathrm{~cm}_{-1}$ and was calculated at $3370 \mathrm{~cm} \_1$ at the same level of theory.18

To identify the most relevant conformation(s) of b-DGalfNAc-OMe, the computed $\mathrm{OH}$ frequencies of the candidate structures are compared with the experimental $\mathrm{OH}$ fingerprint. Note a very important rule of thumb for the comparison of experiment and theory: while a good match is generally obtained for the frequencies, the intensities are more difficult to interpret. In particular, $\mathrm{H}$-bonded modes have a strong intensity in theory, but are broadened and weakened experimentally. In our experimental conditions, H-bonded hydroxyl modes were never observed below $3430 \mathrm{~cm} 1$.

Quite often for less flexible structures - such as b-DGalpNAc-OMe 18 - the spectroscopic analysis confirms that the lowest energy structure is the main, if not the only, conformation present in the gas phase. Here we anticipate that several conformers might be needed to account for the experimental spectrum due to the flexibility of the furanose ring.

Here the lowest energy conformer (SA6) displays two free $\mathrm{OH}$ modes $(\mathrm{O} 5 \mathrm{H}$ and $\mathrm{O} 6 \mathrm{H})$, which match the position of the narrow experimental band at $3660 \mathrm{~cm} \_1$. O3H appears at $3200 \mathrm{~cm} \_1$ and is strongly $\mathrm{H}$-bonded, which justify that it is not observed experimentally. This SA6 structure does not show any mildly $\mathrm{H}$-bonded $\mathrm{OH}$ around 3500 cm_1. Thus the lowest energy conformer matches the main bands of the experimental spectrum, but does not account for all its features. To properly reproduce the IRMPD spectrum, it is essential to take into account one or more higher energy conformers featuring mildly $\mathrm{H}$-bonded $\mathrm{OH}$ modes around $3500 \mathrm{~cm} \mathrm{~cm}_{1}$, such as conformer SA5. Northwest conformers however can be ruled out 
based on their characteristic $\mathrm{OH}$ modes around

3600 cm_1, which are not observed in the
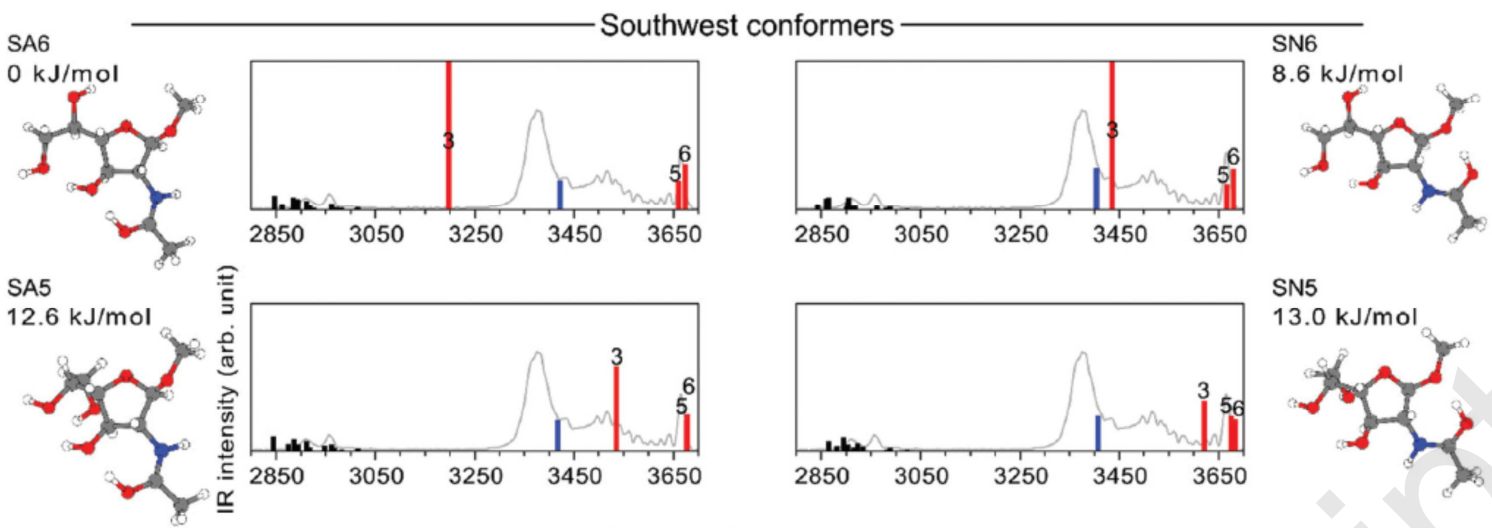

SN5

$13.0 \mathrm{~kJ} / \mathrm{mol}$

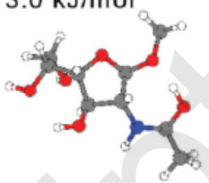

Northwest conformers
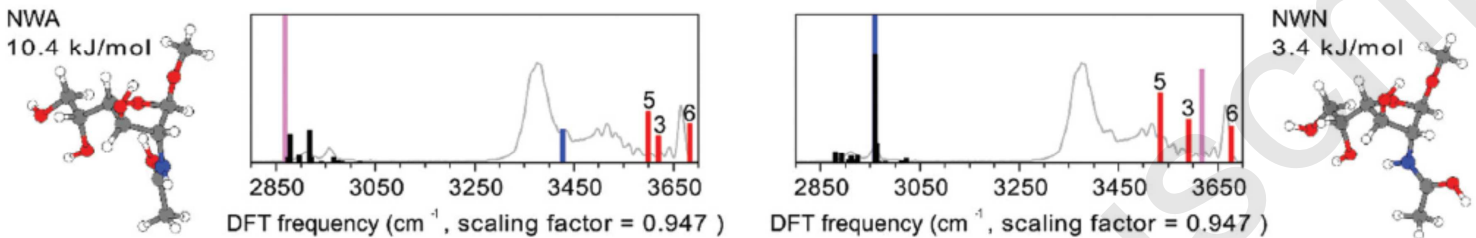

Fig. 5 Experimental spectrum (grey line) compared with typical IR spectra (bars) of 6 conformers of b-D-GalfNAc-OMe 1. A color code is used to identify

the vibrational modes: red (ring $\mathrm{OH})$, blue $(\mathrm{NH})$, black $(\mathrm{CH})$, pink (acetyl $\mathrm{OH}$ ). The labels indicate the ring $\mathrm{OH}$ position.

\section{Conclusions}

In order to correlate structure and function of furanosecontaining glycans, it is critical to develop robust and straightforward analytical approach to detect the carbohydrate sequencing approaches that include the description of the ring size. It is also essential to understand the conformational preferences of furanose rings. Interestingly, IRMPD spectroscopy is a pertinent tool for both the analytical question and the fundamental physical chemistry question.

From an analytical point of view, IRMPD spectroscopy can be used alone (without any computational support) to identify glycans by comparison with a library of pure standards. We have previously demonstrated that the hyphenation ofMS $n$ and IRMPD spectroscopy enables the identification of the monosaccharide content in oligosaccharides, provided reference IRMPD spectra of relevant monosaccharide standards are available.18 To extend this strategy to the description of the ring size, we provide the first spectroscopic signatures of GalNAc in the furanose form. Our results show that the furanose and the pyranose forms feature diagnostic IR fingerprints, which opens the way to the detection of hexofuranosyl residue in oligosaccharides in the case of GalNAc. Other hexofuranosyl residue, such as Galf which is found in bacterial polysaccharides, will be addressed in a future study.

Alternatively, IRMPD spectroscopy can be used in combination with high level ab initio calculations and provides valuable structural information for the understanding of the intrinsic conformational preferences of molecular ions. In this context, we present a comparative assessment of the conformational behavior of the two ring sizes. While GalpNAc has a very simple potential energy surface populated by ${ }_{4} \mathrm{C}_{1}$ conformers, Galf NAc features a much more complex one with low energy structures in both the Northwest and Southwest conformations. This study further suggests that gas phase GalpNAc ions predominantly adopt Northwest conformations. Finally, the coupling of upstream separative methods such as LC 41 or IMS $16,42,43$ with IRMPD analysis would be an interesting prospective of this work: it may enable the analysis of samples where both furanose and pyranose forms coexist.

\section{Conflicts of interest}

There are no conflicts to declare.

\section{Acknowledgements}

Authors thank J. P. Guégan (Ecole Nationale Supe'rieure de Chimie de Rennes, CNRS, UMR 6226) for his help in recording NMR spectra. This work was supported by Institut Universitaire de France, ANR Circe (grant ANR-16-CE30-0012) and the Glycophysics Network (web: hwp://glyms.univlyon1.fr) funded by the French Agence Nationale de la Recherche (grant ANR-2015-MRSEI-0010). This work was granted access to the HPC resources of the FLMSN, "Fédération Lyonnaise de Mode'lisation et Sciences Nume'riques", partner of EQUIPEX EQUIP@MESO and to the "Centre de calcul CC-IN2P3" at Villeurbanne, France.

\section{References}

1 T. L. Lowary, Twenty Years of Mycobacterial Glycans: Furanosides and Beyond, Acc. Chem. Res., 2016, 49, 1379-1388.

2 M. R. M. Hussain, M. Hassan, I. Afzal and A. Afzal, Role of Gal and GalNAc containing glycans in various physiological processes, Egypt. J. Med. Hum. Genet., 2012, 13, 1-9. 
3 T. Ju, V. I. Otto and R. D. Cummings, The Tn Antigen-Structural Simplicity and Biological Complexity, Angew. Chem., Int. Ed., 2011, 50, 1770-1791.

4 J. Nilsson, F. Noborn, A. Gomez Toledo, W. Nasir, C. Sihlbom and G. Larson, Characterization of Glycan Structures of Chondroitin SulfateGlycopeptides Facilitated by Sodium Ion-Pairing and Positive Mode LC-MS/MS, J. Am. Soc. Mass Spectrom., 2017, 28, 229-241.

5 C. Husser, A. Brink, M. Zell, M. B. Müller, E. Koller and S. Schadt, Identification of GalNAcConjugated Antisense Oligonucleotide Metabolites Using an Untargeted and Generic Approach Based on High Resolution Mass Spectrometry, Anal. Chem., 2017, 89, 6821-6826.

6 N. P. Arbatsky, A. S. Shashkov, S. S. Mamyan, Y. A. Knirel, K. Zych and Z. Sidorczyk, Structure of the O-specific polysaccharide of a serologically separate Proteus penneri strain 22, Carbohydr. Res., 1998, 310, 85-90.

7 F. S. Michael, C. M. Szymanski, J. Li, K. H. Chan, N. H. Khieu, S. Larocque, W. W. Wakarchuk, J.-R. Brisson and M. A. Monteiro, The structures of the lipooligosaccharide and capsule polysaccharide of Campylobacter jejuni genome sequenced strain NCTC 11168, Eur. J. Biochem., 2002, 269, 51195136.

8 H. A. Taha, M. R. Richards and T. L. Lowary, Conformational Analysis of Furanoside-Containing Mono- and Oligosaccharides, Chem. Rev., 2013, 113, 1851-1876.

9 M. R. Richards, Y. Bai and T. L. Lowary, Comparison between DFT- and NMR-based conformational analysis of methyl galactofuranosides, Carbohydr. Res., 2013, 374, 103-114.

10 N. C. Polfer and J. Oomens, Vibrational spectroscopy of bare and solvated ionic complexes of biological relevance, Mass Spectrom. Rev., 2009, 28, 468-494.

11 F. O. Talbot and J. P. Simons, Sugars in the gas phase: the spectroscopy and structure of jet-cooled phenyl b-Dglucopyranoside, Phys. Chem. Chem. Phys., 2002, 4, 3562-3565.

12 E. J. Cocinero, E. C. Stanca-Kaposta, M. Dethlefsen, B. Liu, D. P. Gamblin, B. G. Davis and J. P. Simons, Hydration of Sugars in the Gas Phase: Regioselectivity and Conformational Choice in N-Acetyl Glucosamine and Glucose, Chem. Eur. J., 2009, 15, 13427-13434.

13 I. A. Bermejo, I. Usabiaga, I. Companon, J. Castro-Lopez, A. Insausti, J. A. Fernandez, A. Avenoza, J. H. Busto, J. Jimenez-Barbero, J. L. Asensio, J. M. Peregrina, G. Jimenez-Oses, R. Hurtada-Guerrero, E. J. Cocinero and F. Corzana, Water Sculpts the Distinctive Shapes and Dynamics of the Tumor-Associated Carbohydrate Tn Antigens: Implications for Their Molecular Recognition, J. Am. Chem. Soc., 2019, 140, 9952-9960.

14 E. J. Cocinero and P. Çarçabal, in Gas-Phase IR Spectroscopy and Structure of Biological Molecules, ed. A. M. Rijs and J. Oomens, Springer International Publishing, Cham, 2014, vol. 364, pp. 299-333.

15 E. Mucha, A. I. Gonza'lez Flo'rez, M. Marianski, D. A. Thomas, W. Hoffmann, W. B. Struwe, H. S. Hahm, S. Gewinner, W. Scho"llkopf, P. H.
Seeberger, G. von Helden and K. Pagel, Glycan Fingerprinting via Cold-Ion Infrared Spectroscopy, Angew. Chem., Int. Ed., 2017, 56, 11248-11251.

16 C. Masellis, N. Khanal, M. Z. Kamrath, D. E. Clemmer and T. R. Rizzo, Cryogenic Vibrational Spectroscopy Provides Unique Fingerprints for Glycan Identification, J. Am. Soc. Mass Spectrom., 2017, 28, 2217-2222.

17 N. C. Polfer, J. J. Valle, D. T. Moore, J. Oomens, J. R. Eyler and B. Bendiak, Differentiation of Isomers by Wavelength-Tunable Infrared MultiplePhoton Dissociation-Mass Spectrometry: Application to Glucose-Containing Disaccharides, Anal. Chem., 2006, 78, 670-679.

18 L. Barnes, B. Schindler, S. Chambert, A.-R. Allouche and I. Compagnon, Conformational preferences of protonated $\mathrm{N}$-acetylated hexosamines probed by InfraRed Multiple Photon Dissociation (IRMPD) spectroscopy and ab initio calculations, Int. J. Mass Spectrom., 2017, 421, 116-123.

19 B. Schindler, L. Barnes, G. Renois, C. Gray, S. Chambert, S. Fort, S. Flitsch, C. Loison, A.-R. Allouche and I. Compagnon, Anomeric memory of the glycosidic bond upon fragmentation and its consequences for carbohydrate sequencing, Nat. Commun., 2017, 8, 973.

20 C. S. Contreras, N. C. Polfer, J. Oomens, J. D. Steill, B. Bendiak and J. R. Eyler, On the path to glycan conformer identification: Gas-phase study of the anomers of methyl glycosides of $\mathrm{N}$-acetyl-Dglucosamine and N-acetyl-D-galactosamine, Int. J. Mass Spectrom., 2012, 330-332, 285-294.

21 Y. Tan, N. Zhao, J. Liu, P. Li, C. N. Stedwell, L. $\mathrm{Yu}$ and N. C. Polfer, Vibrational Signatures of Isomeric Lithiated N-acetyl-D-hexosamines by GasPhase Infrared Multiple-Photon Dissociation (IRMPD) Spectroscopy, J. Am. Soc. Mass Spectrom., 2017, 26, 539-550.

22 J. Martens, G. Berden, R. E. van Outersterp, L. A. J. Kluijtmans, U. F. Engelke, C. D. M. van Karnebeek, R. A. Wevers and J. Oomens, Molecular identification in metabolomics using infrared ion spectroscopy, Sci. Rep., 2017, 7, 3363.

23 Y. Zhu, L. A. Hamlow, C. C. He, J. K. Lee, J. Gao, G. Berden, J. Oomens and M. T. Rodgers, Gas-Phase Conformations and N-Glycosidic Bond Stabilities of Sodium Cationized 20-Deoxyguanosine and Guanosine: Sodium Cations Preferentially Bind to the Guanine Residue, J. Phys. Chem. B, 2017, 121, 4048-4060.

24 E. J. Cocinero, A. Lesarri, P. E'cija, F. J. Basterretxea, J.-U. Grabow, J. A. Ferna'ndez and F. Castan o, Ribose Found in the Gas Phase, Angew. Chem., Int. Ed., 2012, 51, 3119-3124.

25 E. J. Cocinero, A. Lesarri, P. E'cija, A'. Cimas, B. G. Davis, F. J. Basterretxea, J. A. Ferna'ndez and F. Castaño, Free Fructose Is Conformationally Locked, J. Am. Chem. Soc., 2013, 135, 2845-2852. 26 P. E' cija, I. Uriarte, L. Spada, B. G. Davis, W. Caminati, F. J. Basterretxea, A. Lesarri and E. J. Cocinero, Furanosic forms of sugars: conformational equilibrium of methyl b-D-ribofuranoside, Chem. Commun., 2016, 52, 6241-6244.

27 J. J. P. Stewart, MOPAC2012, 2012.

28 A. D. Becke, Density functional exchange energy approximation with correct asymptotic behavior, 
Phys. Rev. A: At., Mol., Opt. Phys., 1988, 38, 30983100.

29 A. D. Becke, A new mixing of Hartree-Fock and local densityfunctional theories, J. Chem. Phys., 1993, 98, 1372-1377.

30 C. Lee, W. Yang and R. G. Parr, Development of the Colle-Salvetti correlation-energy formula into a functional of the electron density, Phys. Rev. B: Condens. Matter Mater. Phys., 1988, 37, 785.

31 T. Yanai, D. P. Tew and N. C. Handy, A new hybrid exchange correlation functional using the Coulomb attenuating method (CAM-B3LYP), Chem. Phys. Lett., 2004, 393, 51-57.

32 W. J. Hehre, R. Ditchfield and J. A. Pople, SelfConsistent Molecular Orbital Methods. XII. Further Extensions of Gaussian-Type Basis Sets for Use in Molecular Orbital Studies of Organic Molecules, J. Chem. Phys., 1972, 56, 2257-2261.

33 R. Krishnan, J. S. Binkley, R. Seeger and J. A. Pople, Self consistent molecular orbital methods. 20 . basis set for correlated wave functions, J. Chem. Phys., 1980, 72, 650-654.

34 M. J. Frisch, J. A. Pople and J. S. Binkley, Selfconsistent molecular orbital methods 25. Supplementary functions for Gaussian basis sets, J. Chem. Phys., 1984, 80, 3265-3269.

35 C. Moller and M. S. Plesset, Note on an approximation treatment for many-electron systems, Phys. Rev., 1934, 46, 0618-0622.

36 L. Barnes, B. Schindler, A.-R. Allouche, D. Simon, S. Chambert, J. Oomens and I. Compagnon, Anharmonic simulations of the vibrational spectrum of sulfated compounds: application to the glycosaminoglycan fragment glucosamine 6-sulfate, Phys. Chem. Chem. Phys., 2015, 17, 25705-25713.

37 A.-R. Allouche, Gabedit-A graphical user interface for computational chemistry softwares, J. Comput. Chem., 2011, 32, 174-182.

38 M. J. Frisch, G. W. Trucks, H. B. Schlegel, G. E. Scuseria, M. A. Robb, J. R. Cheeseman, G. Scalmani, V. Barone, B. Mennucci, G. A. Petersson, H. Nakatsuji, M. Caricato, X. Li, H. P. Hratchian, A. F. Izmaylov, J. Bloino, G. Zheng, J. L. Sonnenberg, M. Hada, M. Ehara, K. Toyota, R. Fukuda, J. Hasegawa, M. Ishida, T. Nakajima, Y. Honda, O. Kitao, H. Nakai, T. Vreven, J. A. Montgomery Jr., J. E. Peralta, F. Ogliaro, M. J. Bearpark, J. Heyd, E. N. Brothers, K. N. Kudin, V. N. Staroverov, R. Kobayashi, J. Normand, K. Raghavachari, A. P. Rendell, J. C. Burant, S. S. lyengar, J. Tomasi, M. Cossi, N. Rega, N. J. Millam, M. Klene, J. E. Knox, J. B. Cross, V. Bakken, C. Adamo, J. Jaramillo, R. Gomperts, R. E. Stratmann, O. Yazyev, A. J. Austin, R. Cammi, C. Pomelli, J. W. Ochterski, R. L. Martin, K. Morokuma, V. G. Zakrzewski, G. A. Voth, P. Salvador, J. J. Dannenberg, S. Dapprich, A. D. Daniels, O". Farkas, J. B. Foresman, J. V. Ortiz, J. Cioslowski and D. J. Fox, Gaussian 09, Gaussian, Inc., Wallingford, CT, USA, 2009.

39 F. Neese, The ORCA program system, Wiley Interdiscip. Rev.: Comput. Mol. Sci., 2012, 2, 73-78. $40 \mathrm{C}$. Altona andM. Sundaralingam, Conformational analysis of the sugar ring in nucleosides and nucleotides. New description using the concept of pseudorotation, J. Am. Chem. Soc., 1972, 94, 8205-8212.
41 B. Schindler, G. Laloy-Borgna, L. Barnes, A.-R. Allouche, E. Bouju, V. Dugas, C. Demesmay and I. Compagnon, Online Separation and Identification of Isomers Using Infrared Multiple Photon Dissociation Ion Spectroscopy Coupled to Liquid Chromatography: Application to the Analysis of Disaccharides Regio-Isomers and Monosaccharide Anomers, Anal. Chem., 2018, 90, 11741-11745. 42 J. Seo, W. Hoffmann, S. Warnke, X. Huang, S. Gewinner, W. Scho"llkopf, M. T. Bowers, G. von Helden and K. Pagel, An infrared spectroscopy approach to follow b-sheet formation in peptide amyloid assemblies, Nat. Chem., 2017, 9, 39-44.

43 B. Schindler, A. Depraz-Depland, G. RenoisPredelus, G. Karras, B. Concina, G. Celep, J. Maurelli, V. Loriot, E. Constant, R. Bredy, C. Bordas, F. Lepine and I. Compagnon, FAIMS-MS-IR spectroscopy workflow: a multidimensionsl platform for the analysis of molecular isoforms, Int. J. Ion Mobil. Spec., 2017, 20, 119-124. 